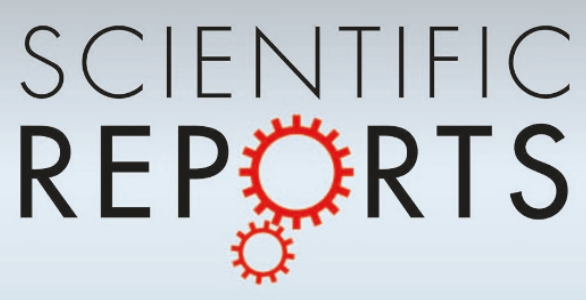

OPEN A novel tissue model for angiogenesis:

SUBJECT AREAS: evaluation of inhibitors or promoters in

CANCER MODELS

TUMOUR ANGIOGENESIS

RECEPTOR PHARMACOLOGY

HIGH-THROUGHPUT SCREENING \title{
tissue level
}

Bingling Dai*, Yanmin Zhang*, Yingzhuan Zhan, Dongdong Zhang, Nan Wang \& Langchong He

School of Medicine, Xi'an Jiaotong University, No. 76, Yanta Weststreet, \#54, Xi'an, Shaanxi Province 710061, P. R. China.

Received

25 June 2013

Accepted

9 December 2013

Published

15 January 2014

Correspondence and requests for materials should be addressed to L.C.H. (helc@mail.xitu. edu.cn)

* These authors contributed equally to this work.
A novel tissue model for angiogenesis (TMA) is established for effective evaluation of angiogenesis inhibitors or promoters in vitro. Lung tissues were cultured in fibrinogen "sandwich" structure which resembled the formation of neovessels in vivo. The cells and capillary-like structures grew from the lung tissues were identified as endothelial cells and neovessels. Both immunohistochemisty and western blot results indicated that autocrine VEGF bound to the KDR and induced KDR autophosphorylation that could induce the proliferation of endothelial cells and their migration as well as the formation of microvessels on the lung tissue edge. With addition of the TMA, the murine VEGF and cultured medium produced by A549 tumor cells apparently promoted the increase of neovessels. Sorafenib as a tumor angiogenesis inhibitor and Tongxinluo as an angiogenesis promoter were both used to evaluate the TMA performance and they exhibited a good effect on neovessels in the TMA. The model established imitated angiogenesis in vivo and could well serve as an effective method in evaluating the angiogenesis inhibitors or promoters, and could also be practical for screening small molecules that affect blood vessel formation.

ngiogenesis is essential to adult tissue remodeling and regeneration, solid tumor growth, and coronary artery disease ${ }^{1}$. During the process of neovascularization, endothelial cell (EC) sprouting from the parent vessel is involved, followed by migration, proliferation, alignment, tube formation, and anastomosis to other vessels ${ }^{2}$. It is a complex process by accurately regulating between pro-angiogenic factors and anti-angiogenic factors ${ }^{3}$. The increasing evidence shows that vascular endothelial growth factor (VEGF) could be a key factor among the large pro-angiogenic factors ${ }^{4}$. It plays an important role in the endothelial cell proliferation and development of angiogenesis-dependent diseases. VEGF binds to its receptor: kinase insert domain containing receptor $(\mathrm{KDR})$ and induces $\mathrm{KDR}$ dimerization, activation and autophosphorylation. Its activation regulates endothelial cell migration, proliferation, differentiation, survival as well as vessel permeability and dilation ${ }^{5}$.

Weibel-Palade body (WPB) is a specialized cigar-shaped secretary organelle in endothelial cells which contains a variety of biologically active molecules. The main component of WPB is a kind of adhesive glycoprotein von Willebrand Factor (vWF), whose expression and tabulation are necessary for the formation of the unique rod-like WPB. VWF is a plasma glycoprotein that circulates in blood as a series of multimersin association with factor VIII procoagulant protein ${ }^{6}$. Meanwhile, CD34, representing microvascular density in the tissue, (human hematopoietic progenitor cell antigen) is selectively expressed on the majority of hematopoietic stem/progenitor cells, bone marrow stromal cells, capillary endothelial cells, embryonic fibroblasts, and some nerve tissues, and is considered to be an important marker of tissue vascularization and represents microvascular density in the tissue ${ }^{7}$. This inspired us to carry out the present study on angiogenesis by using immunofluorescence marker CD34 and vWF for the demonstration of endothelial cells and new microvessels.

Previous studies suggest that the selective destruction of the supporting vasculature of tumors has been proposed as a means of therapy. A number of antiangiogenic agents have been reported to inhibit tumor angiogenesis such as sorafenib which inhibits angiogenesis via inhibition of $\mathrm{KDR}^{9}$. Moreover, blood deficiency in an organ or tissue caused by a constriction or obstruction of its blood vessels is common in organ ischemia. Therefore stimulating angiogenesis is an effective therapy. Tongxinluo Capsule is a traditional Chinese drug used to cure coronary heart disease by promoting angiogenesis ${ }^{10}$. In this study, we adopted them to confirm the effects of TMA in inhibiting and stimulating angiogenesis

Nowadays, various angiogenesis models have been established and applied, including endothelial cells model ${ }^{11}$, endothelial cells co-cultured with tumor cells model ${ }^{12}$, the chick embryo chorioallantoic membrane model ${ }^{13}$, corneal angiogenesis model ${ }^{14}$, matrix culture of rat aorta model ${ }^{15}$ and zebrafish model et $\mathrm{al}^{16}$. Each of them could 
evaluate compounds inhibition on either endothelial cells or vascular sprouting from pre-existing vasculature. But no model at the tissue level to evaluate chemical compounds in the effects of vessel growth has been reported. In this paper, we report the establishment of a novel angiogenesis model at the tissue level in which the process of neovessels imitated the neovascularization in vivo, and, it is useful for studying the molecular mechanisms of angiogenesis inhibitors.

\section{Results}

Established tissue model for angiogenesis. An assay of mouse lung tissue was implanted in the fibrinogen sandwich structure. An assay of cells and capillary-like structures grew out from the edge of the lung tissue (Fig. $1 \mathrm{a}-1 \mathrm{~b}$ ) and grew along with the increase of the culture days. Among these cells, round shape, "cobblestone" shape and spindle shape were noticed under the inverted fluorescence microscope (Fig. 1 a). Analysis of cells under transmission electron microscope demonstrated that the size and shape of the WeibelPalade bodies and their specific internal striations were easily recognizable in the electron microscope (Fig. $1 \mathrm{c}$ ). Indirect immunofluorescence staining further showed that all cells stained with CD34-FITC (green) and cell nuclei stained with DAPI (blue) were considered to be endothelial cells. As shown in Fig. $1 \mathrm{f}$, these CD34positive cells showed "cobblestone" morphology. Subsequently, immunofluorescence staining of capillary-like structures showed the presence of von Willebrand protein in the rodlike structures. As shown in Fig. $1 \mathrm{~h}$, the capillary-like structures were stained with vWF-FITC (green), and the positive results revealed that the capillary-like structures were new microvessels.

In order to investigate the effect of TMA on different conditions, $200 \mu \mathrm{L}$ of five different culture medium (DMEM, RPMI-1640, IMDM, L35, F12) were added to the three-dimensional matrix. No obvious differences of microvessels were observed among the five experimental groups during the cultivation of lung tissue (data not shown). The number of vessels in different groups had no statistical significance. To explore whether the microvessels in the TMA were related to the tumor cell growth, we added the A549 cell cultured supernant to the culture system. The results indicated that microvessels exhibited rapid growth in conditioned medium compared with the control group (Fig. 1 d). A549 tumor cells may secrete something inducing and maintaining the growth vessels.

Mechanism of the TMA. The ELISA assay was used to measure the amount of VEGF secreted during the cultivation of lung tissues. It showed that the quantity of VEGF in supernatant was evidently increased with the increase of the days of culture (Fig. 2 e). The results indicated that the tissues themselves could secrete an important pro-angiogenic factor VEGF in this culture condition which could promote and maintain the growth of vessels.

To explore the changes of CD34, vWF and KDR, p-KDR in the development and angiogenesis of the TMA, the fresh lung tissue and cultured lung tissue (cultured 9 days) were observed with frost slice techniques and immunohistochemical staining. By staining CD34 and $\mathrm{vWF}$, the staining intensity in the cultured tissue ( 9 day) edge was stronger than that of the fresh tissue (Fig. $2 \mathrm{a}_{1}-2 \mathrm{~b}_{3}$ ); meanwhile, the staining intensity of KDR and $\mathrm{p}-\mathrm{KDR}$ was also significantly stronger in the group of the cultured tissue edge compared to that of the fresh tissue (Fig. $2 c_{1}-2 d_{3}$ ).

To further investigate the protein changes during the lung tissue cultured. Western blot assay was used to examine protein expression after the whole lung tissue was cultured. The results showed that the phosphorylation of KDR expression was significantly decreased (Fig. 2 i). CD34 and vWF expression were also reduced (Fig. 2 j).

Then, we added VEGF ( $500 \mathrm{ng} / \mathrm{mL}$ ) to the TMA to further confirm the role of VEGF on this new model. We discovered that with addition of the VEGF, the new microvessels on the periphery of the lung tissues outgrew more than these in the control group (Fig. $2 \mathrm{f}$ ).

Effect of sorafenib and Tongxinluo Capsule on the TMA. We used sorafenib and Tongxinluo Capsule, serving respectively as a tumor
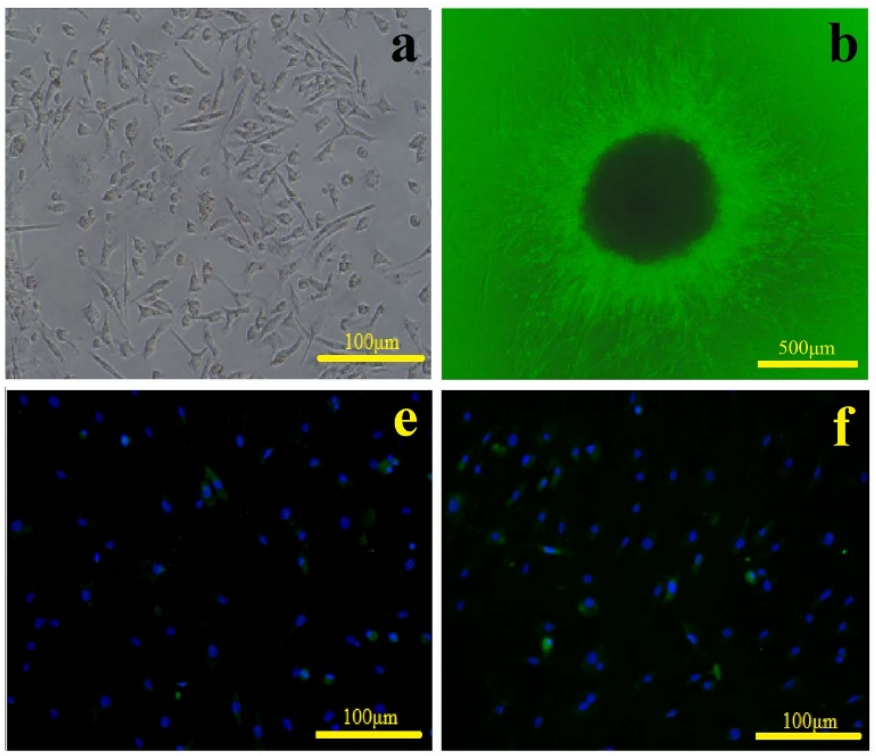
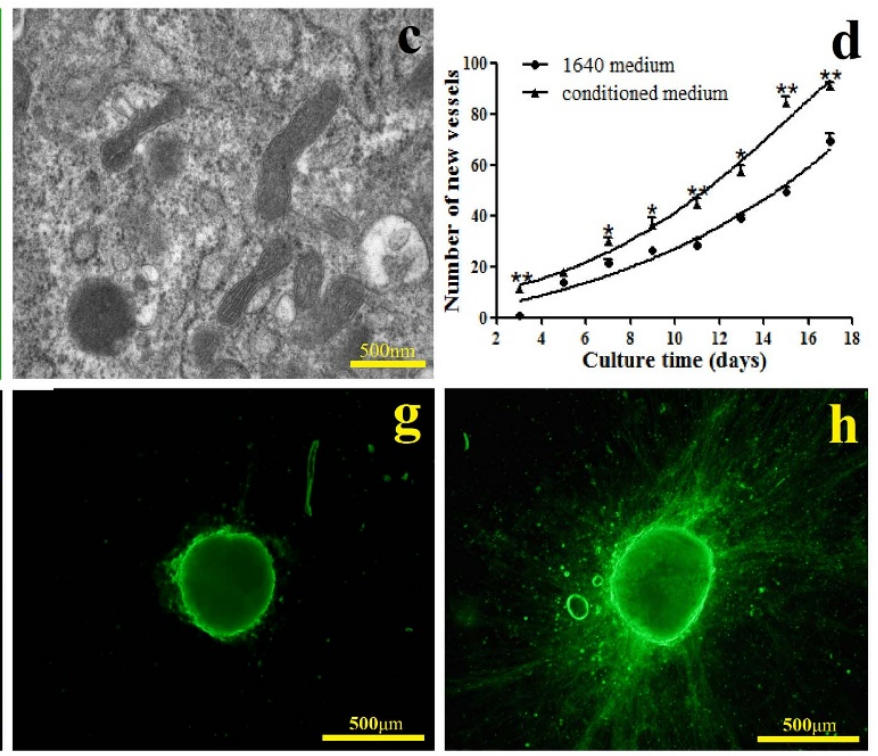

Figure $1 \mid$ Established tissue model for angiogenesis. (a) The image of cells grew from the mouse lung tissue. Arrows were placed to indicate round shape, "cobblestone" shape and spindle shape cells. Bar $=100 \mu \mathrm{m}$. (b) The image of capillary-like structures grew from the lung tissues. Bar $=500 \mu \mathrm{m}$.

(c) Peripheral cells were identified by transmission electron microscopy. The image showed that the electron micrograph of a weibel-palade body which showed typical longitudinal striations. Bar $=500 \mathrm{~nm}$. (d) Effect of conditioned medium on the TMA. A549 cells cultured supernatant was added to the "sandwich" structure. Addition to the conditioned medium, the number of vessels increased quicker compared with vessels in RPMI-1640 medium group. Values are expressed as means \pm SEM. $(\mathrm{n}=4)$. (e) CD34 staining of control. (f) Immunofluorescence micrograph of CD34-stained endothelial cells. The montage image showed the merged fluorescent signal illustrating the human epithelial cells were stained with CD34 marker (green) whereas nuclei were stained with DAPI (blue). Bar $=100 \mu \mathrm{m}$. (g) vWF staining of control. (h) Capillary-like structures were stained with vWF-FITC. The representative image showed that the capillary-like structures were labeled with the vWF (green). Bar $=500 \mu \mathrm{m}$. 

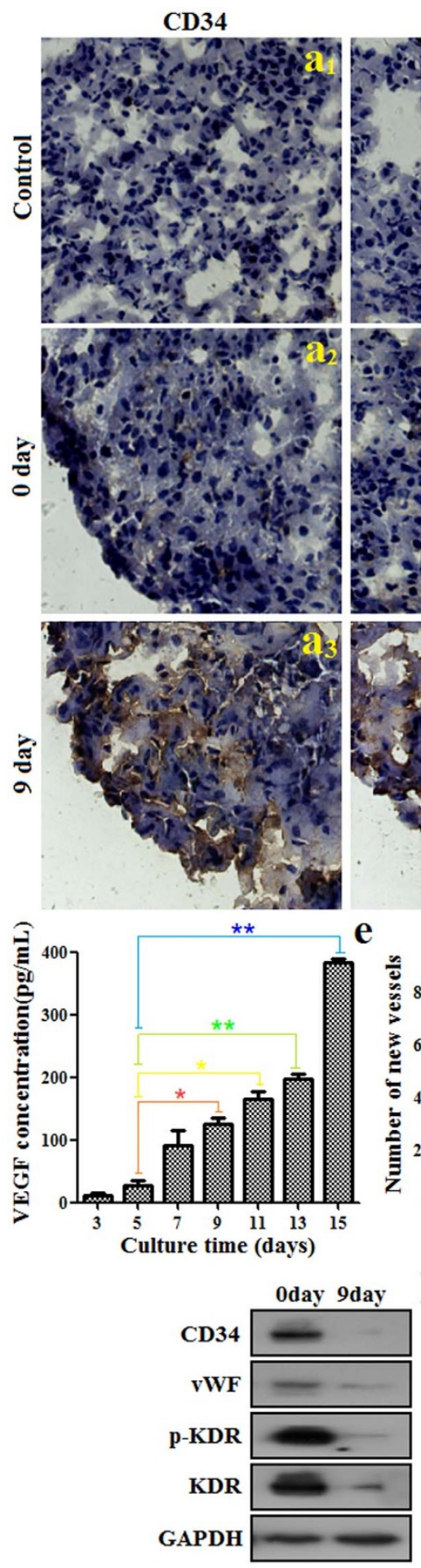

\begin{abstract}
e
\end{abstract}
vWF
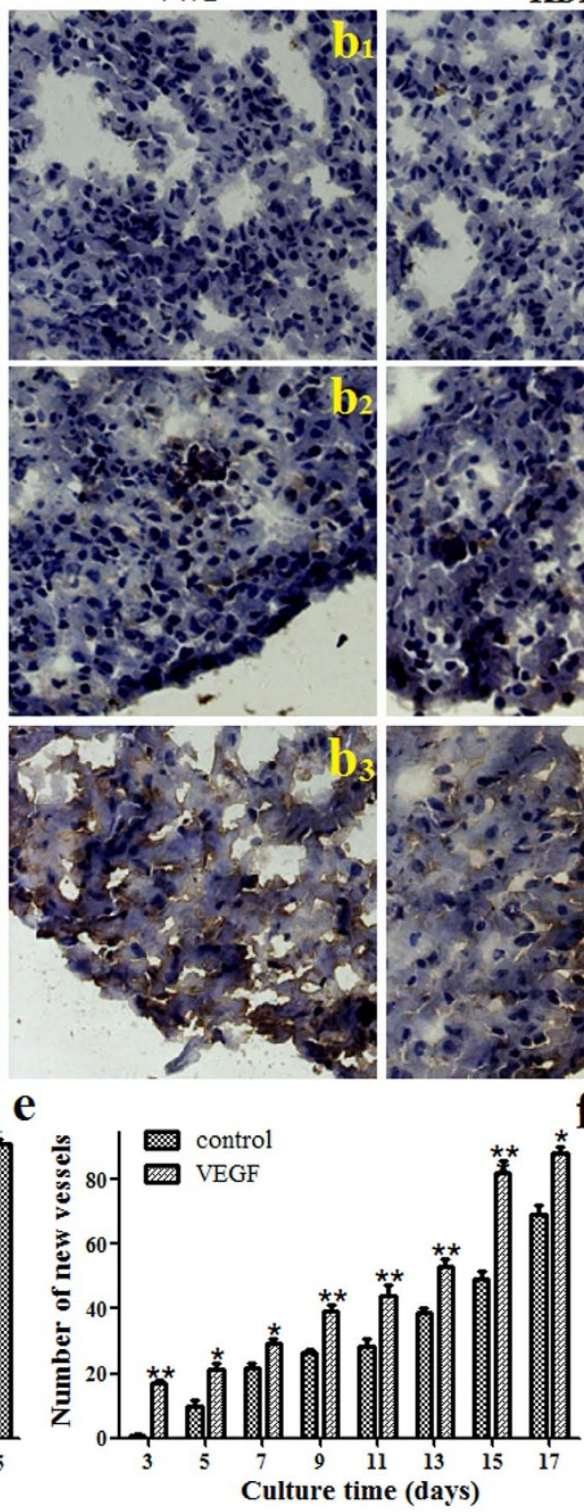

h

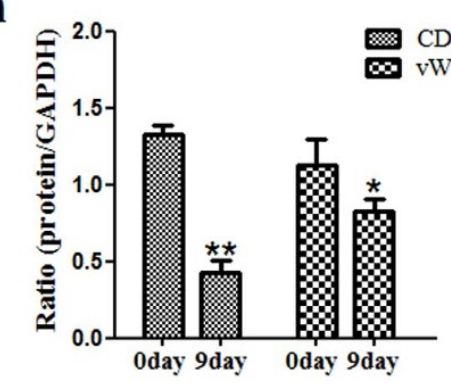

KDR
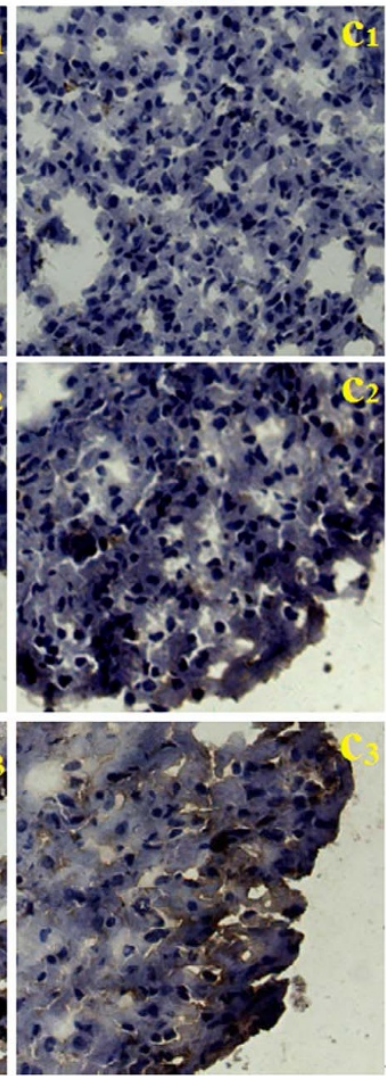

p-KDR
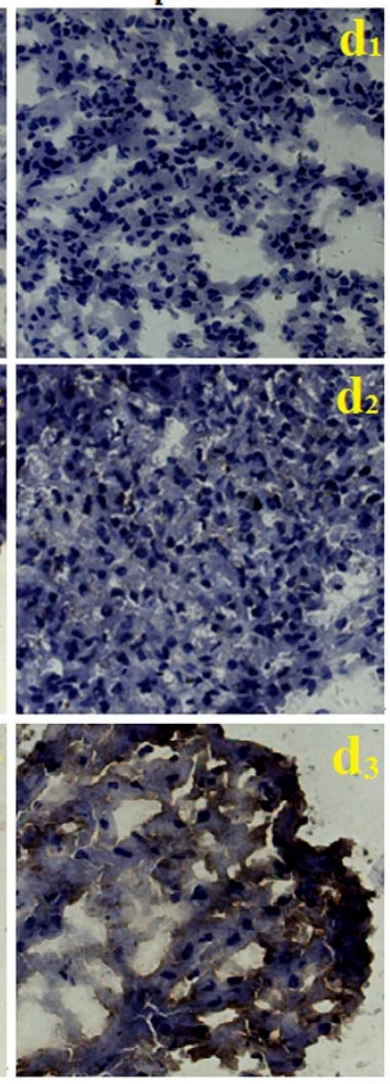

g

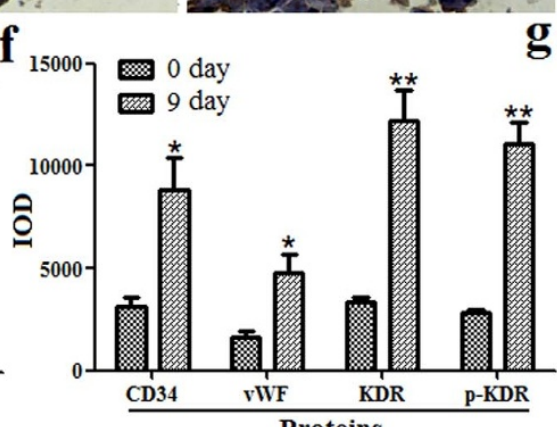

Proteins

i

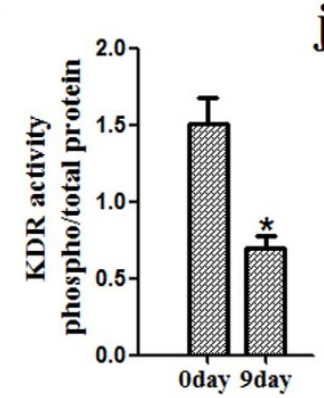

Figure $2 \mid$ Mechanism of the angiogenesis in the TMA. Immunohistochemical staining of CD34, vWF, KDR and p-KDR showed that positive cells labeled brown of fresh lung tissue ( 0 day) and cultured 9 days lung tissue from the TMA. Representative immunostaining pictures are presented ( $\left.\mathrm{a}_{1}-\mathrm{d}_{3}\right)$. Magnification: $200 \times$. (e) Change of VEGF secretion in culture system supernatant. The supernatant was collected every other day and used to quantitative the VEGF. The expression of VEGF in supernatant was evidently increased along with the time went on. Values are expressed as means \pm SEM. $(\mathrm{n}=2) .{ }^{*} \mathrm{P}<0.05,{ }^{* *} \mathrm{P}<0.01$ versus the 5th day group. (f) Effect of VEGF on the TMA. VEGF was added to the culture system, vessels grew quicker than ones in control group. Values are expressed as means \pm SEM. $(\mathrm{n}=4)$. (g) Quantification of protein expression from 4 sections in each group. Data were expressed as mean values $\pm \mathrm{SEM}$. ${ }^{*} \mathrm{P}<0.05,{ }^{* *} \mathrm{P}<0.01$ versus the 0 day group. (h) Western blot analysis of CD34, vWF, KDR and $\mathrm{p}-$ KDR expression in fresh lung tissue ( 0 day) and cultured lung tissues in the TMA (cultured 9 days). Gels were run under the same experimental conditions and GAPDH is shown as a control. The cropped gels images are shown in and the full-length blots/gels are presented in Supplementary Figure 1.

(i) Quantification of CD34 and vWF protein expression. Data were expressed as mean values \pm SEM. $* \mathrm{P}<0.05$, $* * \mathrm{P}<0.01$ versus the 0 day group.

(j) Quantification of $\mathrm{p}-\mathrm{KDR}$ protein expression. Data were expressed as mean values \pm SEM. ${ }^{*} \mathrm{P}<0.05$ versus the 0 day group. 
angiogenesis inhibitor and an angiogenesis promoter, to observe the growth of new vessels. After the lung tissues were implanted onto sandwich structure, tissues were incubated in the absence or presence of sorafenib. As shown in Fig. $3 \mathrm{a}_{1}$, many new microvessels grew in the untreated control group after cultured 9 days. At the same time, with the addition of sorafenib, the new vessels were found to be fewer compared with the cultured tissues without sorafenib (Fig. $3 a_{2}-a_{4}$ ). Sorafenib caused a reduction in new blood vessels growth and destroy the structure of blood vessels compared with the untreated control group. It indicates that sorafenib significantly inhibited microvessels when introduced to the culture system and showed inhibitory in a dose-dependent manner at the concentrations of 20, 100 and $500 \mathrm{nM}$ (Fig. $3 \mathrm{a}_{2}-\mathrm{a}_{4}$ ). The amount and length of vessels gradually decreased along with the culture days prolonged among all groups was shown in Fig. $3 \mathrm{c}-3 \mathrm{~d}$.

Serum contained Tongxinluo Capsule was prepared to act on the TMA. Considering the drug metabolism and excretion, the serum of different times contained different concentrations of drugs. We adopted $1 \mathrm{~h}$ serum, $2 \mathrm{~h}$ serum, $3 \mathrm{~h}$ serum after treatment with Tongxinluo Capsule and mouse serum as control and there were various vessels in different group on 5 th day as shown in Fig. $3 b_{1}-$ $b_{4}$. The results also showed that on the third day the new vessels grew in the tissues in the group of serum while vessels sprouted on the fifth day in the blank group. On the third day, $3 \mathrm{~h}$ serum group grew more vessels compared with the other serum groups. But as shown in Fig. 3 e, $2 \mathrm{~h}$ serum group exhibited the fastest growth rate during the whole cultivation process. Besides, the length and amount of vessels gradually increased along with the culture time prolonged among all groups (Fig. 3 e-3 f).

\section{Discussion}

The main innovation of our study is the establishment of TMA in vitro and exploration of its underlying mechanisms of angiogenesis. In our study, the mouse lung tissues were embedded in fibrinogen to form the three-dimensional "sandwich" structure. During the cultivation, cells and capillary-like structures outgrew from the lung tissues: (1) Under the inverted fluorescence microscope, these cells were shown to be in round shape, "cobblestone" shape and spindle shape; (2) Meanwhile, their microstructures were observed under the transmission electron microscope. Fortunately, the rodlike organelle Weibel-Palade body specifically existed in endothelial cells was detected. We concluded that there were endothelial cells among the peripheral cells; (3) Then, we used immunofluorescence marker CD34 for the identification of peripheral cells. A portion of cells that grew like as "cobblestone" shape was stained with the specific antibody CD34 (green) whereas cell nuclei were stained by DAPI (blue).These results indicated that cells grew as "cobblestone" shape were endothelial cells. This is in line with the results of the previous studies. VWF stored in the Weibel-Palade bodies was specifically associated with new vessels. The specific staining results show that the capillary-like structures were microvessels. All these results indicated that the lung tissues could grow new microvessels in this cultured environment and the model could imitate angiogenesis process in vivo.

Neovascularization is accompanied with a process that includes the activation of resting endothelial cells, endothelial cell proliferation, tube formation, and eventually differentiation into new vessels. An assay of experiment was carried out to assess the procedure in the TMA. VEGF recognized as one of the important proangiogenic factors was used to determine whether the new vessels respond to this positive angiogenic influences ${ }^{17}$. In our study, results from the ELISA showed that the amount of VEGF in supernatant accumulated more and more along with the culture time prolonged (in $\mathrm{pg} / \mathrm{mL}$ range). We can infer that the lung tissue itself secreted VEGF in this culture environment. Then we added murine VEGF (in $\mathrm{ng} / \mathrm{mL}$ range) to the cultured system to further verify the role of VEGF in the TMA. The results showed that more vessels can be induced in VEGF-added group than in blank group. All the results indicated that the new vessels responded to VEGF which was confirmed as a known proangiogenic factor and VEGF played a critical role in the growth of new microvessels in the TMA.

The autocrine VEGF binding to KDR and induces KDR autophosphorylation of specific tyrosine residues within the dimeric complex. KDR activation further regulates the endothelial cell proliferation and migration. Even though CD34 is commonly used as an endothelial cell marker, its expression is not restricted to endothelium, which also is expressed on hemopoietic stem cell et $\mathrm{al}^{7}$. On this basis, we evaluated the amount of endothelial cells with CD34 and vWF. The immunohistochemical analysis indicated that the $\mathrm{CD}_{3} 4^{+}$and $\mathrm{vWF}^{+}$cells obviously increased in lung tissues edge of the TMA. Meanwhile, western blot results showed that CD34 and vWF expression were reduced. Both immunohistochemical analysis and western blot elucidated that activated endothelial cells migrated from inner lung tissue to the edge. Increase of phosphorylation KDR in the edge of lung tissue implied that the process of angiogensis occurred on the edge of lung tissue. Our results elucidated the underlying mechanism of angiogenesis in the TMA was that: after the lung tissue was cut and cultured in the fibrinogen structure, endothelial cells which were located in the inner lung tissue responded to the secreted VEGF and the culture system. Then endothelial cells proliferation and gradually migrated to the tissue edge and formed new blood vessels from the tissue edge into the fibrinogen "sandwich" structure. The mechanisms of angiogenesis were similar with that of the tumor angiogenesis.

Solid tumor growth depends on angiogenesis that supplied oxygen and nutrients ${ }^{18}$; in turn, tumor cell released growth factor to promote angiogenesis. In this TMA, different culture mediums showed no different effects on the angiogenesis, but the A549 tumor cell cultured medium promoted the vessels growth. We could infer that A549 tumor cells secretion promoted the growth of the vessels. This could offer a basis for further study on the relation between the tumor cell and the TMA.

To evaluate the TMA, we used Tongxinluo Capsule and sorafenib that inhibit angiogenesis via inhibiting KDR, and they could respectively stimulate and inhibit angiogenesis clinically ${ }^{10}$. We obtained the serum containing Tongxinluo Capsule by serum pharmacology method which could reflect the actual concentration of Tongxinluo Capsule in vivo. Exposed to Tongxinluo Capsule, the new vessels grew more and quicker in the serum than in the blank group. A continuous treatment of $20 \mathrm{nM}, 100 \mathrm{nM}$, and $500 \mathrm{nM}$ of sorafenib evidently inhibited the growth of the vessels compared with the untreated group. The number and length of vessels were less in the sorafenib-treated group and reduced in a dose-dependent manner. These clinic drugs exhibited their pharmacological activity on the model we established. Recent efforts in developing new treatment options to aberrant angiogenesis and neovascular destruction of various organs have aimed at targeting the new vessels. The model was exactly confirmed to meet the requirement of screening effective compounds on vessel growth and then provides a theoretical basis for further development.

There are currently several angiogenesis models for evaluating the growth of the vessels which involves endothelial cells model ${ }^{11}$ and endothelial cells co-cultured with tumor cells model in cell level ${ }^{12}$, the chick embryo chorioallantoic membrane model $^{13}$, corneal angiogenesis model ${ }^{14}$, matrix culture of rat aorta model $^{15}$ and zebrufish model $^{16}$ in vivo and in vitro. Traditional endothelial cells model is experimentally simple, but it evaluated by cells proliferation, migration and tube formation separately. In 3D culture of cell lines assay, it supply a three dimensional spatial structure, but endothelial cells finally formed luminal net structure not vessels. Corneal angiogenesis model is experimentally elaborate and expensive so that it is not for rapid drug screening. Chick embryo chorioallantoic membrane 

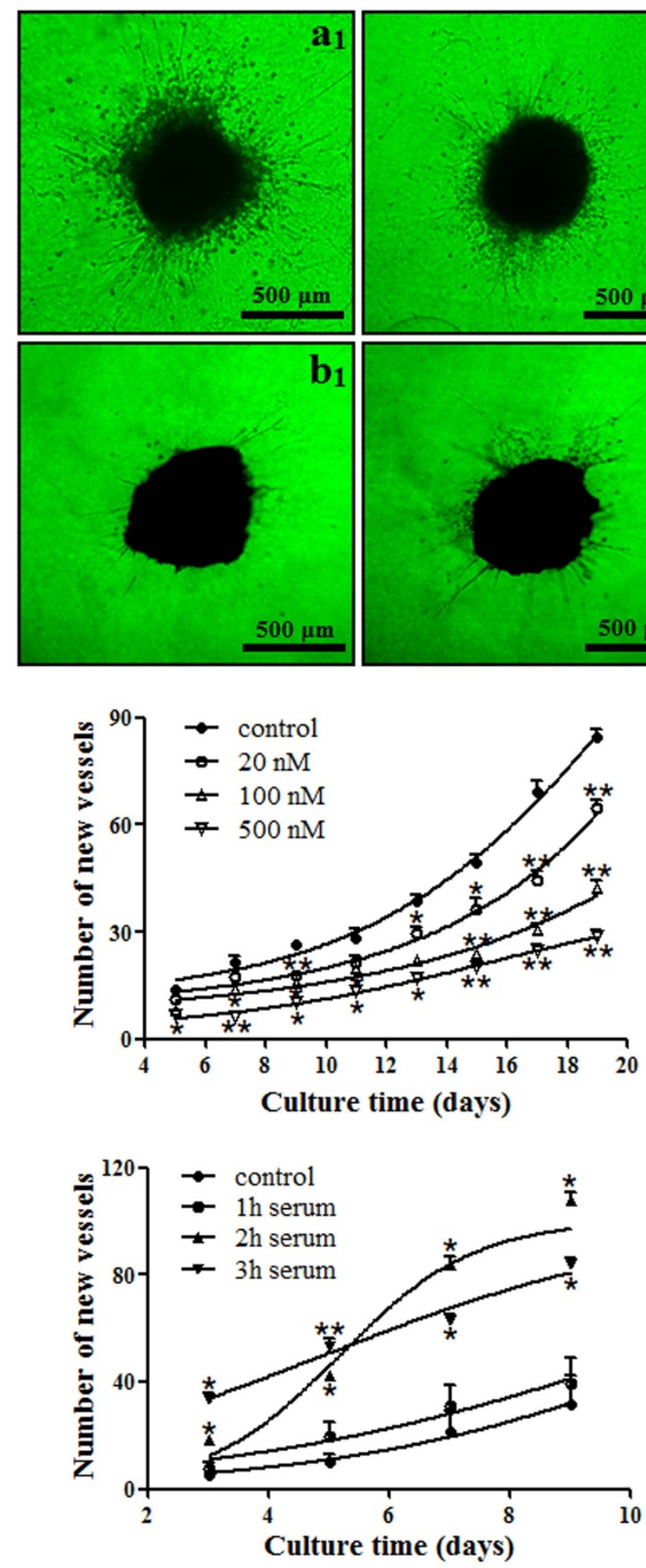
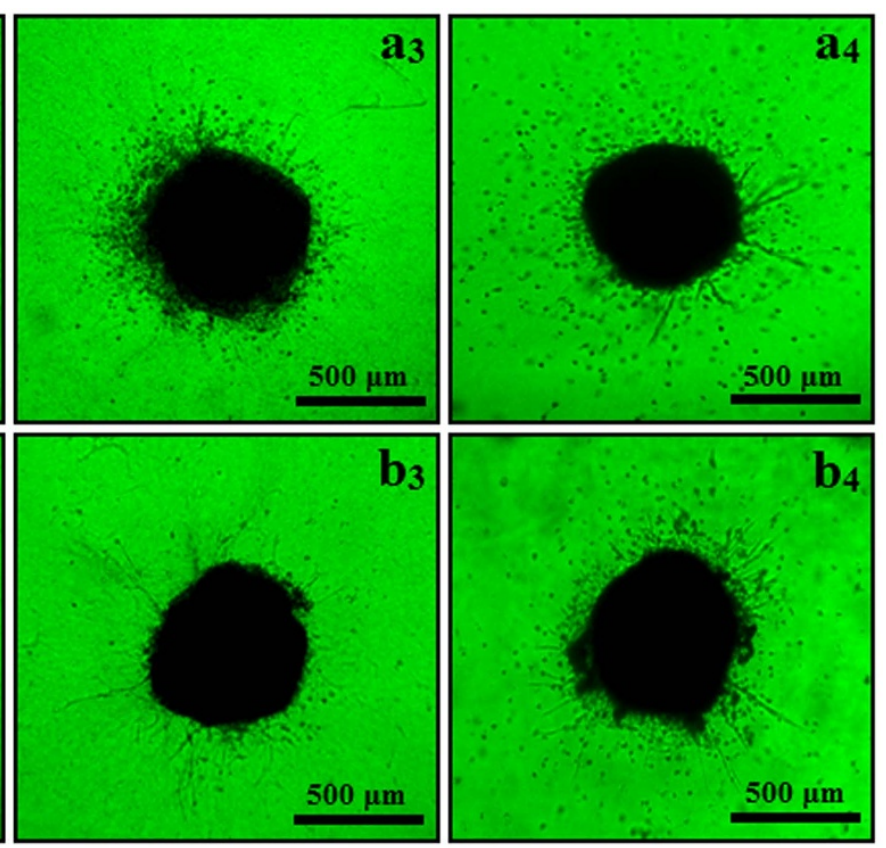

c
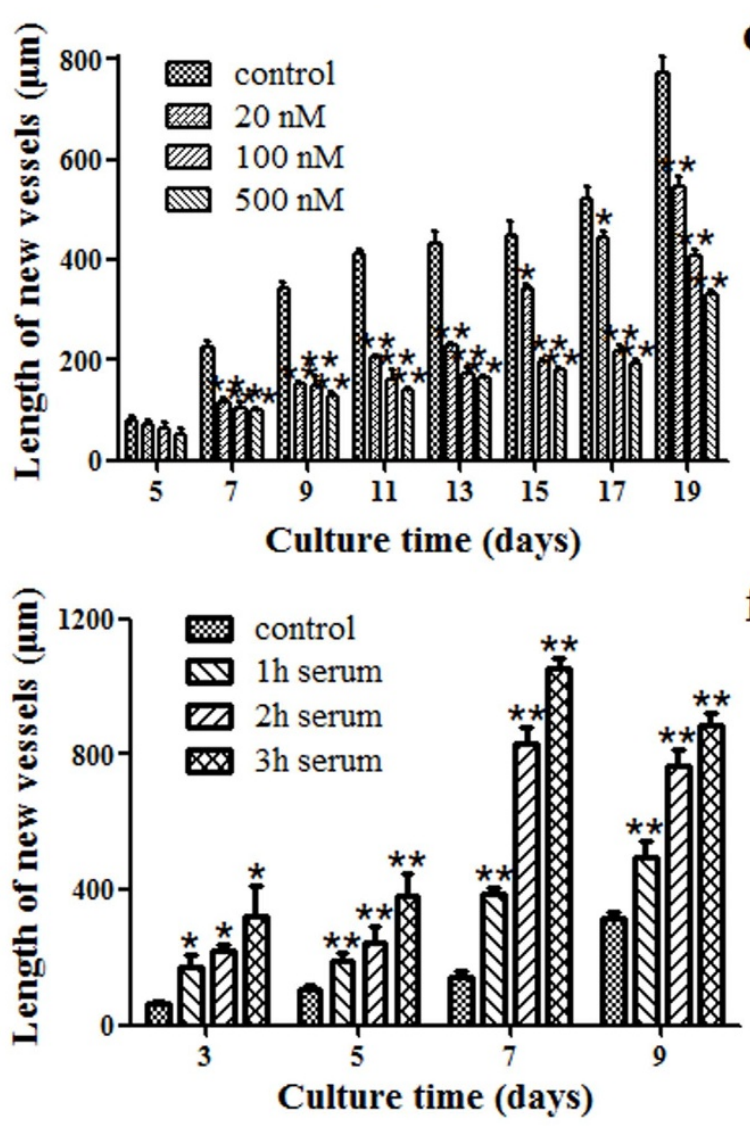

Figure 3 Sorafenib inhibits angiogenesis and Tongxinluo capsule promotes angiogenesis in the TMA. $\left(a_{1}\right)$ The representative images of lung tissue blood vessels in the untreated group on the 9th day; $a_{2}-a_{4}$ : lung tissue vessels in the sorafenib-treated group on the 9 th day; $\left(a_{2}\right) 20 \mathrm{nM},\left(a_{3}\right) 100 \mathrm{nM},\left(a_{4}\right)$ $500 \mathrm{nM}$. Vessels grew normally in control group; vessels in the sorafenib $(20,100,500 \mathrm{nM})$-treated group exhibited the slowly increase compared with the control group. Bar $=500 \mu \mathrm{m}$. $\left(\mathrm{b}_{1}\right)$ The representative images of lung tissue blood vessels in the untreated group on the 5 th day; $b_{2}-b_{4}$ : lung tissue vessels in the sorafenib-treated group on the 9th day; $\left(b_{2}\right) 1 \mathrm{~h}$ serum, $\left(b_{3}\right) 2 \mathrm{~h}$ serum, $\left(\mathrm{b}_{4}\right) 3 \mathrm{~h}$ serum. (c) Quantification of microvessels numbers in sorafenib treated group with the increased days. $(n=3)(d)$ Quantification of vessels length in sorafenib treated group along with the time prolonged. Data were expressed as mean values \pm SEM. $(\mathrm{n}=7)$. ${ }^{*} P<0.05,{ }^{* *} P<0.01$ versus the control group. (e) Quantification of microvessels numbers in Tongxinluo capsule treated group with the increased days. $(\mathrm{n}=3)(\mathrm{f})$ Quantification of vessels length in Tongxinluo capsule treated group along with the time prolonged. Data were expressed as mean values \pm SEM. $(\mathrm{n}=7)$. ${ }^{*} P<0.05,{ }^{* *} P<0.01$ versus the control group. 


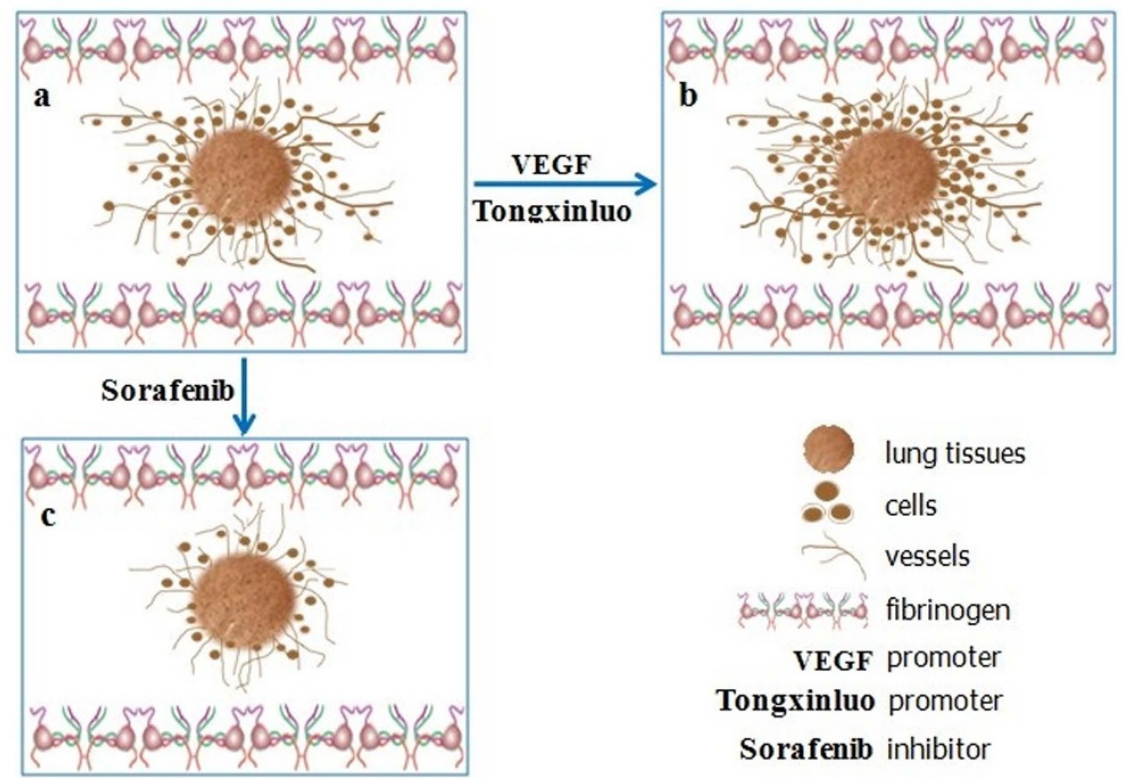

Figure $4 \mid$ Scheme of tissue model for angiogenesis (TMA). (a) The cells and vessels sprout from the lung tissues in the cultured medium like as "sandwich" structure. (b) VEGF or Tongxinluo promotes angiogenesis of TMA. (c) Sorafenib inhibits angiogenesis of TMA.

model and zebrufish model both have been used to screen, however, there are several limitations in assays of screening. Assays in both models need a lot of embryo with a big cost, and the chick embryo requires a culture that should be conducted in a sterile environment. In the previous study, Han, RNN et a $\mathrm{l}^{19}$ cultured animal and human lung explants which were taken from $12 \mathrm{wk}$ gestation fetuses and used CD34 immunostaining to observe the number of endothelial cells with or without IGF-1. Their results reflected morphologic changes of human fetal lung explants during 5 days cultivation and validated the role of IGF-1 in vasculogenesis/angiogenesis. But in the literature, there was no evidence on angiogenesis process of lung explants. This also provided scientific basis for our study. The main advantages of the TMA compared with existing ones are as follows. For one thing, the experiment is simple operation and this method provided multiple data points from each animal. Meanwhile, each assay was determined for three times using different animals to provide a valid result and the assay presented good repeatability. For another, the cultured lung tissues in three dimensional environments simultaneously involve the endothelial cells proliferation, migration and the formation of new microvessels. It maximizes the process of angiogenesis in vivo compared with 3D culture of rat aorta and 3D culture of cell line. Furthermore, the lung tissue taken from the mouse was cut into small pieces $\left(0.5-1 \mathrm{~mm}^{3}\right)$, so we could harvest an abundance of explants from each animal. It is practical for screening a large number of compounds simultaneously using a microplate.

All together, we successfully established a novel tissue model for angiogenesis. As depicted in Fig. 4, the endothelial cells migrated from the cultured lung tissue periphery and finally differentiated into new vessels. Its underlying mechanism is that the autocrine VEGF stimulates KDR expression and the VEGF binding to KDR induces autophosphorylation of VEGF in the TMA. This model provides a useful method in rapid evaluating and screening of novel angiogenesis inhibitors and promoters.

\section{Methods}

Reagents. DMEM, RPMI-1640, IMDM, L35, F12 were purchased from GIBCO (USA). Trypsin was from Amresco (USA). Matrigel was obtained from BD Biosciences (Franklin Lakes, NJ, USA). Fibrinogen from bovine plasma, DAPI was from Sigma (USA). Thrombin was obtained from Guoao Pharmaceutical (Changchun, China). KDR and p-KDR monoclonal antibody were from Cell Signaling Technology. CD34 Polyclonal Antibody was from Santa Cruz Biotechnology (USA). vWF Polyclonal Antibody, rabbit-anti-GAPDH and
FITC-Goat Anti-Rabbit IgG ( $\mathrm{H}+\mathrm{L})$ were purchased from Protein technology Group (USA). Antifade mounting medium was from Beyotime biotechnology (Shanghai, China). Enzyme-linked Immunosorbent Assay (ELISA) kits were purchased from R\&D Systems (Minneapolis, MN, USA). Murine VEGF was from PeproTech (USA). Tongxinluo capsule was purchased from Yiling Pharmaceutical (Shijiazhuang, China). Tissue-Tek O.C.T. Compound was from SAKURA (USA). HistostainTMPlus Kits and DAB kit were purchased from ZSGB-BIO (Beijing, China).

Cell culture and mice. Human non-small cell lung cancer cell line A549 was purchased from Shanghai Institute of Cell Biology in the Chinese Academy of Sciences (Shanghai, China). A549 cells were cultured in RPMI-1640 supplemented with $10 \% \mathrm{FBS}$ and incubated at $37^{\circ} \mathrm{C}$ in a $5 \% \mathrm{CO}_{2}$ atmosphere.

Mice (15-18 g), SD rats (200-220 g) were purchased from Animal Research center of Xi'an Jiao tong University. The mice and rats were maintained under laminar air flow conditions with a 12-h light (6:00-18:00)/12-h dark (18:00-6:00) cycle.

Laboratory food and water were freely available. Animal care was in accordance with the National Institute of Health guidelines and the Animal Research Committee of Xi'an Jiao tong University.

A statement identifying the institutional and/or licensing committee experimental approval. All animal experiments were carried out according to the guidelines and approval of the Institutional Animal Care and use Committee of Xi'an Jiao tong University.

The culture of mouse lung tissue. The mouse (4-6 weeks old, body wt. 15-18 g) was soaked in $75 \%$ alcohol for $5 \mathrm{~min}$ after it was sacrificed. Then a complete autopsy was performed. The lung tissue was immediately taken out and dipped into sterile PBS to wash for three times. Next it was cut into small pieces $\left(0.5-1 \mathrm{~mm}^{3}\right)$ quickly. Then an assay of pieces was placed into a layer of previous lypolymerized fibrinogen with thrombin in 48-well plate. Afterwards, fibrin matrices with thrombin were put on the tissue to form a second gel layer. After consolidation, $200 \mu \mathrm{L} /$ well $10 \%$ FBScontaining 1640 medium was added. The 48 -well plate was incubated at the conditions of $37^{\circ} \mathrm{C}$ and $5 \% \mathrm{CO}_{2}$. Meanwhile, the lung tissue was monitored and photographed every day.

Indentification of endothelial cells and neovessels. The peripheral cells of the lung tissue were harvested and fixed at $4{ }^{\circ} \mathrm{C}$ in glutaraldehyde overnight. Then the cells were washed with PBS, post-fixed in $1 \%$ phosphate-buffered $\mathrm{OsO}_{4}$ for $60 \mathrm{~min}$, dehydrated in serial alcohol solutions and embedded in epoxy resins. Ultrathin sections $(50-70 \mathrm{~nm})$ on grids were stained for $20 \mathrm{~min}$ with aqueous uranyl acetate and for $3 \mathrm{~min}$ in lead citrate. Specimens were examined on a TEM (Hitachi, Japan).

The peripheral cells were seeded at a density of $1 \times 10^{5}$ cells on the cover slips, grown to confluence. For CD34 immunostaining, the cover slips were washed three times with PBS and fixed in $4 \%$ paraformaldehyde for $15 \mathrm{~min}$ at R.T. The nonspecific interaction was blocked with 3\% BSA (in PBS) at R.T for $1 \mathrm{~h}$. The cells were incubated with monoclonal rabbit CD34 ( $1: 100$ dilution) antibody at $4{ }^{\circ} \mathrm{C}$ overnight. Then the cells were incubated with FITC-goat anti rabbit IgG ( $1: 100$ dilution) at R.T for $1 \mathrm{~h}$ in dark. After being rinsed in PBS, the slips were incubated with DAPI $(1: 1000$ dilution) for $5 \mathrm{~min}$ at R.T. The capillary-like structures were performed following the same way with the peripheral cells. The vWF polyclonal antibody was 1: 50 dilution 
and FITC-goat anti rabbit IgG was 1:100 dilution. Images were then captured under the inverted fluorescence microscope. Incubation without the primary antibody was performed as a control for the background staining.

Different conditions on the TMA. The lung tissues were cultured into a sandwich structure of lypolymerized fibrinogen with thrombin as described previously in 48well plates. Firstly, we explored the effect of different culture mediums on the TMA. $200 \mu \mathrm{L}$ of DMEM, RPMI-1640, IMDM, L35 and F12 containing 10\% FBS were added to individual wells for culturing the lung tissues. Secondly, $200 \mu \mathrm{L}$ of fresh conditioned medium (A549 cells cultured supernatant fluid) was added to the "sandwich" structure every other day and RPMI-1640 medium was added as the control. Again, murine VEGF (500 ng/mL) was added to the culture system and the same amount of solvent as control. Images were captured under the inverted fluorescence microscope and the growth rate of vessels was analyzed by counting the number of vessels.

The amount of VEGF in the culture system was measured in duplicate by ELISA analyses performed following the manufacturer's instructions. The concentrations of VEGF $(\mathrm{pg} / \mathrm{mL})$ were calculated from a standard curve using commercial reagents and a standard protocol.

Immunohistochemical staining of the lung tissue. The cultured lung tissue (cultured for 9 days) in the new model was taken and fixed in liquid nitrogen for $24 \mathrm{~h}$ and stored at $-80^{\circ} \mathrm{C}$. The fresh lung tissue was used as a control. The tissue was embedded within OCT and the frozen sections. $10 \mu \mathrm{m}$-frozen sections of tissues were fixed in $4 \%$ paraformaldehyde for $15 \mathrm{~min}$ at R.T. Then the sections were stored at $-80^{\circ} \mathrm{C}$ and used for immunostaining with anti-CD34, anti-vWF, anti-KDR and antiphosphorylated KDR,under the manufacturer's instructions. Incubation without the primary antibody was performed as a control for the background staining. Three sections per lung tissue were analyzed at $200 \times$ magnification. For quantitative analysis, the ImagePro Plus analysis system was used to measure $\mathrm{CD} 34^{+}, \mathrm{vWF}^{+}$, $\mathrm{KDR}^{+}, \mathrm{p}-\mathrm{KDR}^{+}$areas in each random fields of per section.

Western blot analyze. The cultured lung tissue (cultured for 9 days) in the new model was taken from the culture system and the fresh lung tissue was harvested. Then the tissue were lysed with RIPA lysis Buffer supplemented with protease inhibitor cocktail tablets and phosphatase inhibitor cocktail tablets on ice for $30 \mathrm{~min}$. The lysates was removed by centrifugation at $12,000 \mathrm{rpm}$ for $10 \mathrm{~min}$ at $4^{\circ} \mathrm{C}$. Protein concentration was determined by the BCA Protein Quantification kit according to manufacturer's instructions. The cell lysates were denatured by boiling with $5 \times$ reducing sample buffer for $5 \mathrm{~min}$ and equivalent amount of protein was run on SDSPAGE gel. After electrophoresis, separated proteins were transferred to polyvinylidene difluoride membrane and blocked with 5\% non-fat milk in Trisbuffered saline Tween-20 (TBST) buffer for $2 \mathrm{~h}$ at room temperature with continuous agitation. Membranes were then incubated with specific primary antibodies included anti-KDR, anti-p-KDR ( $1: 500$ dilution), anti-CD34, anti-vWF, anti-GAPDH ( $1: 1000$ dilution) overnight at $4^{\circ} \mathrm{C}$ followed by washing and incubated with secondary antibodies at dilution of $1: 20000$ in TBST buffer for $2 \mathrm{~h}$ at $37^{\circ} \mathrm{C}$. The membranes were then washed with TBST buffer for $10 \mathrm{~min}$ for 3 times and developed with enhanced chemiluminescence (ECL) kit.

Sorafenib and Tongxinluo capsule applied to the angiogenesis model. Stocked concentration of sorafenib $(20 \mathrm{mmol} / \mathrm{L})$ was prepared with dimethyl sulfoxide (DMSO) and stored at $4^{\circ} \mathrm{C}$. Different concentrations of the sorafenib diluted in $10 \%$ FBS-containing 1640 medium were added to a 48 -well plate where lung tissue was cultured.

Serum Pharmacology was adopted to obtain the serum containing Tongxinluo ${ }^{20}$. The content of Tongxinluo Capsule was prepared for $44.6 \mathrm{mg} / \mathrm{mL}$ with distilled water (ten times the amount of the adult). Then rats were randomly grouped into the control group (distilled water group) and the treatment (Tongxinluo Capsule) group $(\mathrm{n}=3$ per group). Animals were administrated orally with Tongxinluo solution at the dose of $10 \mathrm{~mL} / \mathrm{kg}$, whereas the control animals received equivalent volumes of dissolvent. The same dose of drugs was administrated orally $2 \mathrm{~h}$ later in the same way. Then after $1 \mathrm{~h}, 2 \mathrm{~h}, 3 \mathrm{~h}$ of twice treatment with drugs or vehicle, the blood was obtained germ freely from orbital venous plexus. The serum was acquired by centrifugation at $12000 \mathrm{rpm}$ for $5 \mathrm{~min}$. After filtration with $0.22 \mu \mathrm{m}$ cellulose acetate membrane, the serum was bottled. Next, the serum was inactivated by $56^{\circ} \mathrm{C}$ water for $30 \mathrm{~min}$ and then added to the TMA.

Respective images were photographed every other day under the inverted fluorescence microscope. We counted the newly formed microvessels and used image analysis software (NIS-elements) to measure the length and the abundance of these sprouting vessel $\mathrm{s}^{21}$

Statistical analysis. All values were expressed as mean \pm SEM (standard error of the mean). The statistical software SPSS 18.0 was used to analyze statistical data and ANOVA was used to analyze the differences between the groups. A P-value $<0.05$ was considered statistically significant.
1. Carmeliet, P. Mechanisms of angiogenesis and arteriogenesis. Nat Med 6, 389-395 (2000).

2. Folkman, J. Tumor angiogenesis: a possible control point in tumor growth. Ann Intern Med 82, 96-100 (1975).

3. Carney, D. N. Lung cancer-time to move on from chemotherapy. New Engl J Med 346, 126-128 (2002).

4. Ferrara, N., Gerber, H. P. \& LeCouter, J. The biology of VEGF and its receptors. Nat Med 9, 669-676 (2003)

5. Ferrara, N. \& Kerbel, R. Angiogenesis as a therapeutic target. Nature 438, 967-974 (2005).

6. Giblin, J. P., Hewlett, L. J. \& Hannah, M. J. Basal secretion of von Willebrand factor from human endothelial cells. Blood 112, 957-964 (2008).

7. Asahara, T. Isolation of putative progenitor endothelial cells for angiogenesis. Science 275, 964-967 (1997).

8. Folkman, J. et al. Tumor angiogenesis: therapeutic implications. N Engl J Med 285 , $1182-1186$ (1971).

9. Jeanny, B. \& Aragon-Ching, W. L. Dahut Anti-angiogenesis approach to genitourinary cancer treatment. Update Cancer Ther 3, 182-188 (2009).

10. Yu, Y. H., Xu, X. Q., Wang, Y., Sun, S. Z. \& Chen, Y. Intervention of Tongxinluo capsule against vascular lesion of atherosclerosis and its effect on lectin-like oxidized low density lipoprotein receptor-1 expression in rabbits. Chin J Integr Med 12, 32-36 (2006).

11. Shyu, K. G., Tsai, S. C., Wang, B. W., Liu, Y. C. \& lee, C. C. Saikosaponin C induces endothelial cells growth, migration and capillary tube formation. Life Sci 76, 813-826 (2004).

12. Kehers, F., Lewalle, J. M., Desreux, J., Munaut, C., Laetitia, D. \& Foidart, J. M. Induction endothelial cell apoptosis by solid tumor cells. Exp Cell Res $\mathbf{2 4 0}$, 197-205 (1998)

13. Zhang, Y. M., He, L. C., Meng, L., Luo, W. J. \& Xu, X. M. Suppression of tumorinduced angiogenesis by taspine isolated from Radix et Rhizoma Leonticis and its mechanism of action in vitro. Cancer lett 262, 103-113 (2008).

14. Conrad, T. J., Chandler, D. B., Corless, J. M. \& Klintworth, G. K. In vivo measurement of corneal angiogenesis with video data acquisition and computerized image analysis. Lab Invest 70, 426-434 (1994).

15. Nicosia, R. F. \& Ottinetti, A. Growth of microvessels in serum-free matrix culture of rat aorta: a quantitative assay of angiogenesis in vitro. Lab Invest 63, 115-122 (1990).

16. Serbedzija, G. N., Flynn, E. \& Willett, C. E. Zebrufish angiogenesis: a new model for drug screening. Angiogenesis 3, 353-359 (1999).

17. Zhang, Y. M. et al. A novel angiogenesis inhibitor impairs lovo cell survival via targeting against human VEGFR and its signaling pathway of phosphorylation. Cell Death Dis 3, 1-9 (2012).

18. Folkman, J. Anti-angiogenesis: a new concept for therapy of solid tumors. Ann Surg 175, 409-416 (1972).

19. Han, R. N. N., Post, M., Tanswell, A. K. \& Lye, S. J. Insulin-like growth factor-I receptor-mediated vasculogenesis/angiogenesis in human lung development. Am J Respir Cell Mol Biol. 28, 159-69 (2003).

20. Wang, B. C., Zhu, L. C. \& Chen, Q. Primary study on the application of Serum Pharmacology in Chinese traditional medicine. Colloids Surfaces B 43, 194-197 (2005).

21. Nicosia, R. F. \& Ottinetti, A. Growth of microvessels in serum-free matrix culture of rat aorta: a quantitative assay of angiogenesis in vitro. Lab Invest. 63, 115-122 (1990).

\section{Acknowledgments}

This work was supported by National Natural Science Foundation of China (Grant 81001447,81227802 and 81370088)

\section{Author contributions}

B.L.D. and Y.M.Z. designed and performed the research, analysed the data and wrote the manuscript. Y.Z.Z., D.D.Z. and N.W. performed some of the experiments. L.C.H. supervised the project reviewed the manuscript.

\section{Additional information}

Supplementary information accompanies this paper at http://www.nature.com/ scientificreports

Competing financial interests: The authors declare no competing financial interests.

How to cite this article: Dai, B.L. et al. A novel tissue model for angiogenesis: evaluation of inhibitors or promoters in tissue level. Sci. Rep. 4, 3693; DOI:10.1038/srep03693 (2014).

This work is licensed under a Creative Commons Attribution-
NonCommercial-ShareAlike 3.0 Unported license. To view a copy of this license, visit http://creativecommons.org/licenses/by-nc-sa/3.0 